\title{
Primary Malignant Melanoma of the Vagina without Metastasis: A Rare Case and Review of Literature
}

\author{
1Department of Radiology, National Institute of Oncology, UHC Ibn \\ Sina, Mohamed V. University, Rabat, Morocco \\ ${ }^{2}$ Department of Oncology, National Institute of Oncology, UHC Ibn \\ Sina, Mohamed V. University, Rabat, Morocco \\ ${ }^{3}$ Department of Radiology, Central Radiology, UHC Ibn Sina, \\ Mohamed V. University, Rabat, Morocco
}

Wilson Bizimana ${ }^{1}$ Gloria Akimana² Arthur Semedo Insumbo ${ }^{3}$ Hounayda Jerguigue

\begin{abstract}
Address for correspondence Wilson Bizimana, MD, Department of Radiology, National Institute of Oncology, UHC Ibn Sina, Mohamed V. University, Rabat 8093, Morocco (e-mail: wilson.bizimana@gmail.com).
\end{abstract}

Asian J Oncol:2021;7:45-48

\begin{abstract}
Keywords

- malignant melanoma

- vagina

- imaging features

Malignant melanoma of vagina is a rare condition. Its histogenesis has been debated and the positive diagnosis is based on immunohistochemistry. Pelvic magnetic resonance imaging remains the gold standard for assessing locoregional extension status and post-treatment surveillance of melanoma of vagina. The observation concerned a 53-year-old woman with no specific history who presented a primary malignant melanoma of vagina without secondary locations. To date, the case is the second one reported in the literature. Early diagnosis of the malignant melanoma of vagina may improve patient survival because late diagnoses are punctuated by poor prognosis. We have presented epidemiological with etiopathogenic characteristics and described all imaging features to stage the tumor and to conduct the appropriate treatment.
\end{abstract}

\section{Introduction}

Primary malignant melanoma of the vagina (PMMV) is a rare malignancy with a poor prognosis. It has a notoriously aggressive behavior with a high risk to local recurrence and distant metastasis. ${ }^{1}$ Malignant melanoma accounts for $<10 \%$ of all female genital tract melanomas, for 2.4 to $2.8 \%$ of all vaginal malignancies, and for 0.3 to $1 \%$ of all malignant melanomas. ${ }^{2}$ This neoplasm usually occurs between the sixth and the seventh decade. ${ }^{3}$ The more common symptoms are bleeding or vaginal discharge, a vaginal mass felt, dyspareunia, pruritus, or pain. ${ }^{4}$ No staging system has been demonstrated to be a useful predictor of prognosis in vaginal melanoma, although tumor size $(<3 \mathrm{~cm}$ vs. $\geq 3 \mathrm{~cm}$ ) has been shown to predict survival in some series. ${ }^{5}$ The American Joint Committee on Cancer staging system in vaginal melanoma proposed standardization system in which we distinguish stages I, II, and III

published online December 13, 2020
DOI https://doi.org/ $10.1055 / \mathrm{s}-0040-1722110$ ISSN 2454-6798. corresponding to clinically localized disease, regional lymph node involvement, and distant metastases, respectively. ${ }^{5,6}$ The objective of this study is to discuss epidemiological and etiopathogenic factors, clinical presentation, and finally the diagnosis difficulty of this rare entity.

\section{Patients and Observations}

A 53-year-old female without specific history, G1P1, was admitted to the Gynecological Oncology Department with a chief complaint of vaginal bleeding of unknown cause for 4 months. This sign was associated with vaginal discharge in the form of unspecified leukorrhea mimicking hydrorrhea and reported that a mass was sometimes coming out of her private parts when setting. At inspection, bilateral labia major and minor and clitoris were normal except that they seemed to be elevated. Clinical examination in the speculum (c) 2020. Spring Hope Cancer Foundation \& Young Oncologist Group of Asia.

This is an open access article published by Thieme under the terms of the Creative Commons Attribution-NonDerivative-NonCommercial-License, permitting copying and reproduction so long as the original work is given appropriate credit. Contents may not be used for commercial purposes, or adapted, remixed, transformed or built upon. (https://creativecommons.org/licenses/by-nc-nd/4.0/).

Thieme Medical and Scientific Publishers Pvt. Ltd. A-12, 2nd Floor, Sector 2, Noida-201301 UP, India 
showed a bulging ulcerating mass at the left lateral wall of the vagina limited to two-thirds of the upper vagina measuring approximately $2.5 \mathrm{~cm}$ in diameter. There were no abnormalities of uterus cervix or vulva and there was not palpable node at physical examination. His attending physician ended examination with biopsy and sent reports to the pathologist. Magnetic resonance imaging (MRI) of pelvic was performed and revealed an intravaginal well-limited tissue process, lateralized to the left in T1 hyposignal, T2 intermediate signal, diffusion hypersignal, and enhanced heterogeneously by the injection of gadolinium. Its volume was $18 \times 19 \times 30$ $\mathrm{mm}$ or $5 \mathrm{~g}$ in weight. On the topographic plan, laterally, the tumor bulged opposite to the left of puborectal muscle without touching the parameters. Above, the tumor remained at a distance from the cervix and resected the vaginal sac ipsilaterally. At the bottom, the tumor infiltrated the upper two-thirds of the vagina. In the front and the back, the tumor remained confined to the vaginal wall. We noticed a left external iliac node of $6 \mathrm{~mm}$ diameter. We did not find pelvic or lomboaortic nodes. We concluded to vaginal malignant process lateralized to the left of the upper two-thirds with external iliac node nonspecific. The anatomopathological report of the biopsy revealed that the contaminated sample contained eight fragments weighing $2 \mathrm{~g}$. They measured between 0.7 and $2.3 \mathrm{~cm}$ along their longest axis. The histological study showed a malignant tumor characterized by a diffuse cellular layer made up of large elements provided with a cytoplasm of variable abundance. The nuclei were clearly enlarged, rounded, or oval, rarely irregular, and always hyperchromatic with prominent nucleoli with some atypical mitoses. In addition, there were significant melanic deposits ( - Fig. 1). Thoracoabdominal CT and cerebral CT did not find secondary lesions at a distance. Finally, PMMV without secondary lesion was confirmed. The patient underwent conservative surgery (partial colpectomy) with the removal of the single node. There are no particularities about operating suites.

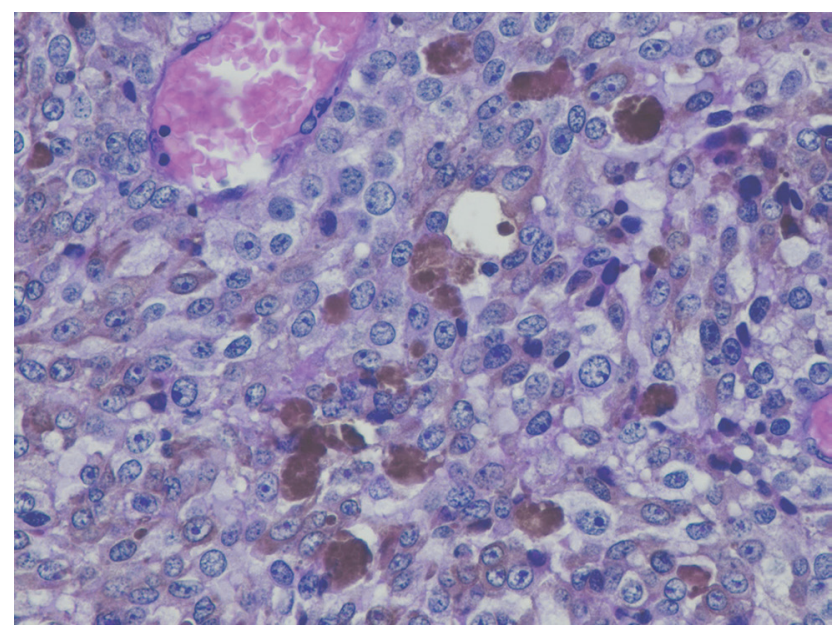

Fig. 1 Microscopic image of blade from anatomopathologist. Malignant tumor formation characterized by a diffuse cellular layer made up of a large element provided with a cytoplasm of variable abundance and contained significant melanic deposits: malignant melanoma.

\section{Discussion}

Malignant melanomas are generally found in areas of skin exposed to the sun, but nonexposed sites, such as genital tract and esophagus could be of concern. ${ }^{7}$ The PMMV is a very rare tumor. It was first reported in 1887 and only almost 306 cases have been reported to date. Its annual incidence is 0.026 per 100,000 women and 0.46 per one million women. ${ }^{6}$ The disease is encountered between 37 and 72 years of age with an average of 57 years. In approximately $73 \%$ of the cases women were menopausal and older than 60 years. ${ }^{8}$ The incidence rate has been found to be very high for postmenopausal women, ranging between $2.08 / 100,000$ for 60 - to 69 -year-old and $4.43 / 100,000$ for 80 - to 89 -yearold women. ${ }^{6}$

The vaginal melanoma is most often located in the lower third (58\%), and in the anterior wall $(45 \%)$ of the vagina. ${ }^{4}$ About $20 \%$ of the mucosal melanomas are multifocal, and only 10 to $23 \%$ are amelanotic melanoma. Epithelioid is the most common histological cell type of PMMV (55\%). Other less common histological cell types of PMMV are spindled (17\%) and mixed (28\%) cells. ${ }^{8}$ Melanocytes have been obtained embryologically from the neural crest. During migration to the epidermis, certain melanocytes could remain aberrantly in the vaginal mucosa or in the endocervical canal in 3\% of these women. They will then be the cause of primary vaginal, cervical, or vulvar melanomas. ${ }^{9,10}$ The diagnosis was often made at an advanced stage as the vagina is characterized by a very rich lymphatic network, considering the high risk of hematogenous extension of melanoma. ${ }^{3}$

Currently, the mechanism of pathogenesis and etiological factors for PMMV are unknown. Socioeconomic factors such as level of education, income, and poverty seem to have no influence on incidence rates and race was not a risk factor for primary malignant vaginal melanoma. ${ }^{6}$ The molecular biology of these melanomas also differs from cutaneous melanomas, with BRAF being rarely seen (seen in almost $70 \%$ cases of cutaneous melanomas). There is also a possible implication of hormonal influence (puberty, pregnancy, menopause, and the use of oral contraceptives) and HPV in melanomas. ${ }^{11}$ Recurrent genital infection, vaginal hysterectomy scar with repeated sexual trauma, immunity deficit as melanoma of vagina was antigenic tumor, family predisposition, and local precancerous lesions were the risk factors discussed in literature. ${ }^{6}$ There were theories which proposed that KIT gene mutations which encode for a protein called c-KIT and are microenvironmental in nature are cited in pathogenesis and there is no relation with ultraviolet radiation. ${ }^{6,8}$

The most common symptoms and signs in patients with PMMV are vaginal bleeding (80\%), vaginal discharge (25\%), palpable vaginal mass (15\%), and pain (10\%). ${ }^{12}$ Clinically, the tumor may be uni- or multifocal, sometimes pigmented and commence from the vulva and end upward at the uterine cervix. ${ }^{6,13}$ This tumor usually occurs in the anterior wall of the vagina, typically in the lower one-third. However, there are other case reports suggesting that the tumor can also arise in the upper third as it is described in our case. ${ }^{6}$ 
Upon visual inspection, the tumor may appear nodular (pigmented mass of brown, blue, or black) or polypoid (polyp-like) in nature. The tumor usually bleeds on touch and is ulcerated in many patients. ${ }^{6}$ The differential diagnoses in front of a small, well-defined, single vaginal mass include metastasis from other sites, poorly differentiated squamous cell carcinoma, superinfected Gartner cyst, epidermoid inclusion cysts, sarcoma, lymphoma and blue nevus. ${ }^{14}$

MRI is the adjuvant useful in lesional exploration in the initial diagnosis. It specifies the seat of the lesion, the size, the limits, its locoregional relations, and the enhancement after injection of gadolinium. ${ }^{13}$ According to our case, on MRI the tumor had T1 hyposignal, T2 hypersignal, with restriction of diffusion and presented an early enhancement after injection of the contrast medium. MRI characteristics of melanoma have been discussed in the context of metastatic intracranial melanoma and melanomas involving the eye in which the predominant MRI signs were hyperintensity on T1-weighted images and hypointensity on T2-weighted images but the signal could be increased in some cases in $\mathrm{T} 2$ and the mass enhanced moderately after injection. These findings were attributed to either the paramagnetism of the stable free radicals occurring in melanin or the methemoglobin in hemorrhagic regions within the tumor. ${ }^{15}$ In short, malignant melanomas may have different signal characteristics depending on melanin concentration and stage of hemorrhage.A malignant melanoma should be considered when a vaginal mass unusually presents high signal intensity on T1-weighted MR image..$^{15}$ The remote extension assessment includes a thoracoabdominopelvic and cerebral CT because metastasis may be localized on brain, liver, lungs, and ganglionic level. PET (positron emission tomography) scan plays a role in the search for and confirming metastases. ${ }^{16} \mathrm{CT}$ of the pelvis showed an ovoid high-attenuation mass of vagina. ${ }^{15}$ CT scan helped also to search for pelvic or paraaortic lymph node needed to improve classification. The confirmation of diagnosis is histological with the use of immunohistochemical study. ${ }^{2}$ However, amelanotic appearance is rare and makes diagnosis even more difficult. Consequently, immunochemical staining should often be used to supplement the diagnosis, such as protein S-100, HMB-45, Melan-A, Mart-1, and vimentin. ${ }^{8}$ We did not find local or distant metastasis or suspects nodes except left external iliac node which was nonspecific. Its early diagnosis and size $<3 \mathrm{~cm}$ could explain the absence of secondary localization.

For staging, the use of FIGO (International Federation of Gynecology and Obstetrics) and TNM (tumor node metastasis) classification is recommended but the prognosis of melanomas also depends on the thickness of the tumor according to the Breslow index, depth invasion, and ganglionic status. ${ }^{13}$

There is no standard treatment for PMMV established in the literature, but surgical excision either by local wide excision or radical surgery with colpectomy with/without exenteration is the mainstay of treatment for women with PMMV., ${ }^{3,417}$ Radiotherapy may have role either as an adjuvant following surgery or when surgery is not feasible due to the disease or patient-related factors. ${ }^{5,11}$ Surgery, radiotherapy, and immunotherapy with interferon- $\alpha$ are the mainstay of treatment for localized disease, while chemotherapy with dacarbazine is mostly used for unresectable and recurrent disease. ${ }^{4,17}$ Let us know that our case did not present secondary lesions which earned him his unusual presentation.The disease is associated with a high risk of local recurrence, distant metastases, and poor clinical outcome. ${ }^{1}$ Studies report that the mean duration of recurrence-free survival was short,i.e., 16 months and the mean overall survival time was only 22 months. ${ }^{17}$

\section{Conclusion}

PMMV is a rare malignancy with a poor prognosis whose incidence is always rising. The tumor is most often diagnosed in late-stage menopausal women with metastasis. We emphasize that our case did not present secondary lesions which earned him his unusual presentation.

\section{Authors' Contributions}

W.B. wrote the manuscript and organized images. G.A. contributed to microscopic image search and proofreading of the manuscript. A.I.S. proofread the manuscript. H.J. reviewed and approved the manuscript. Y.O. did the conceptual input, manuscript reviewing, and final approval of the manuscript. R.L. supervised and approved the final manuscript.

\section{Conflict of Interest}

None declared.

\section{Acknowledgments}

The authors thank Prof. Fouad Kettani of United Nations Center for Pathological Anatomy and his center for their contribution in the availability of ANAPATH block and microscopic image of blade.

\section{References}

1 Tanwar RK, Saxena B, Ali S, Saxena S. Primary malignant melanoma of the vagina: report of two rare cases. J Cancer Res Ther 2018;14(6):1439-1441

2 Chen L, Xiong Y, Wang H, Liang L, Shang H, Yan X. Malignant melanoma of the vagina: a case report and review of the literature. Oncol Lett 2014;8(4):1585-1588

3 Sellal N, Haddad H, Benchakroun N, et al. Mélanome malin primitif du vagin: à propos d'un cas et revue de la littérature. Imag Femme 2011;21:76-79

4 Daix M, Dillies P, Gueuning A, Watkins-Masters L, Gielen F. Mélanome vaginal primitif. Rev Med Liege 2018;73(7-8): 413-418

5 Guler Y, Irem O, Cagdas Y, et al. Primary malignant melanoma of the vagina: report of two cases and review of the literature. J Clin Med Case Rep 2014;1(2):35-41

6 Kalampokas E, Kalampokas T, Damaskos C. Primary vaginal melanoma, a rare and aggressive entity. a case report and review of the literature. In Vivo 2017;31(1):133-139

7 Venkatraman J, Govindaraj T, Rathna S. Primary vaginal melanoma-a rare case report. Sch J App Med Sci 2017;5(2D): 570-573

8 Lalzuitluanga CJ, Kishalay B, Yengkhom IS, et al. Primary malignant melanoma of the vagina: a rare case report. J Dent Med Sci 2019;18(12):68-70 
9 Bennani A, el Fatemi H, Erraghay S, et al. Le mélanome primitif de la muqueuse génitale féminine: à propos de trois observations et revue de littérature. Pan Afr Med J 2013;16:58

10 Androutsopoulos G, Terzakis E, Ioannidou G, et al. Vaginal primary malignant melanoma: a rare and aggressive tumor. Case Rep Obstet Gynecol 2013;2013:137908

11 Rath S, Nanda SS, Singh AP, et al. Aggressive metastatic malignant melanoma of vagina: a case report. J Mol Oncol Res 2018; 2(1):3-5

12 Wohlmuth C, Wohlmuth-Wieser I, May T, Vicus D, Gien LT, Laframboise S. Malignant melanoma of the vulva and vagina: a US population-based study of 1863 patients. Am J Clin Dermatol 2020;21(2):285-295

13 Asaggau S, Badraoui MB, Zouita B, et al. Primary vaginal melanoma in a young woman. J Oncopathol Clin Res 2018;2(2):6
14 Pankaj S, Kumari A, Nazneen S, Choudhary V, Kumari S. Malignant melanoma of vagina: a report and review of literature. J Obstet Gynaecol India 2016;66(5):394-396

15 Woo KM, Seung HK, Hyeong JJ, et al. Malignant melanoma of the vagina: CT and MR findings. J Korean Radiol Soc 1993; 29(3):497-500

16 Nai GA, Bazan A, Rocha CA, Nagy JS, Campos IT. Postpartum genital melanoma-a case report. Rev Bras Ginecol Obstet 2018;40(3):163-167

17 Rapi V, Dogan A, Schultheis B, Hartmann F, Rezniczek GA, Tempfer CB. Melanoma of the vagina: case report and systematic review of the literature. Anticancer Res 2017;37(12): 6911-6920 